\title{
Changes in myocardial cytoskeletal intermediate filaments and myocyte contractile dysfunction in dilated cardiomyopathy: an in vivo study in humans
}

\author{
S Di Somma, M Marotta, G Salvatore, G Cudemo, G Cuda, F De Vivo, M P Di Benedetto,
} F Ciaramella, G Caputo, $\mathrm{O}$ de Divitiis

\begin{abstract}
Aim-To investigate in vivo the intermediate cytoskeletal filaments desmin and vimentin in myocardial tissues from patients with dilated cardiomyopathy, and to determine whether alterations in these proteins are associated with impaired contractility.

Methods-Endomyocardial biopsies were performed in 12 patients with dilated cardiomyopathy and in 12 controls (six women with breast cancer before anthracycline chemotherapy and six male donors for heart transplantation). Biopsy specimens were analysed by light microscopy and immunochemistry (desmin, vimentin). Myocyte contractile protein function was evaluated by the actin-myosin in vitro motility assay. Left ventricular ejection fraction was assessed by echocardiography and radionuclide ventriculography.

Results-Patients with dilated cardiomyopathy had a greater cardiomyocyte diameter than controls $(\mathrm{p}<0.01)$. The increase in cell size was associated with a reduction in contractile function, as assessed by actin-myosin motility $(r=-0.643$; $\mathrm{p}<0.01)$. Quantitative immunochemistry showed increased desmin and vimentin contents $(\mathrm{p}<0.01)$, and the desmin distribution was disturbed in cardiomyopathy. There was a linear relation between desmin distribution and actin-myosin sliding in vitro $(r=0.853 ; \mathrm{p}<0.01)$ and an inverse correlation between desmin content and ejection fraction $(r=-0.773 ; \mathrm{p}<0.02)$. Negative correlations were also found between myocardial vimentin content and the actin-myosin sliding rate $(r=-0.74 ; \mathrm{p}<0.02)$ and left ventricular ejection fraction $(r=-0.68 ; \mathrm{p}<0.01)$.

Conclusions-Compared with normal individuals, the myocardial tissue of patients with dilated cardiomyopathy shows alterations of cytoskeletal intermediate filament distribution and content associated with reduced myocyte contraction.

(Heart 2000;84:659-667)
\end{abstract}

Department of

Experimental and

Clinical Medicine,

University Federico II,

via Pansini 5, 80131

Naples, Italy

S Di Somma

M Marotta

G Salvatore

G Cudemo

M P Di Benedetto

G Caputo

$\mathrm{O}$ de Divitiis

Department of Clinical and Experimental Medicine, University of Magnor Graecia, Catanzaro, Italy G Cuda

Department of Clinical and Experimental Medicine, Second University of Naples, Italy

F Ciaramella

Cardiac Surgery

Department, Second University of Naples F De Vivo

Correspondence to: Dr Di Somma cardio@uol.it

Accepted 16 August 2000

Keywords: dilated cardiomyopathy; desmin; vimentin; cardiac biopsy; actin-myosin

Myocardial alterations in the human heart affected by dilated cardiomyopathy include myocyte loss, "slippage" of myocytes within the wall, segmental replacement, and interstitial fibrosis with hypertrophy of residual myocytes. ${ }^{1}$ These histological changes are non-specific and are similar to those in patients with heart failure from other causes, such as coronary heart disease, hypertension, or valve diseases. ${ }^{2}{ }^{3}$

The cytoskeleton of myocardial cells is a well organised structure, with a complex array of proteins that maintains the internal organisation of cellular organelles and transmits the mechanical forces within the cell to and from the adjacent cells and extracellular matrix. ${ }^{4}$ It is composed of microtubules, microfilaments, and intermediate filaments, such as desmin and vimentin. ${ }^{5}$

It has recently been suggested that the cytoskeleton, together with the contractile proteins and the excitation-contraction coupling mechanisms, represents a major determinant of the intrinsic function of the myocyte and that this could be the primary site of cellular damage in the clinical syndrome of heart failure. ${ }^{67}$ Desmin and titin act as the major stress bearing elements in the sarcomere, ${ }^{8}$ and an increase in desmin content has been described in myocyte hypertrophy. ${ }^{9}$ Moreover, in the transition from compensated ventricular hypertrophy to heart failure, Collins and colleagues have shown a progressive increase in myocyte desmin levels. ${ }^{8}$ To elucidate the pathophysiology of dilated cardiomyopathy further, Schaper and associates investigated the changes in the cytoskeleton and associated proteins and found increased amounts of desmin, tubulin, vinculin, and vimentin; they concluded that these morphological changes were "the structural correlates of the reduced function in chronic cardiac failure." ${ }^{\prime 0}$ An increase in myocardial cytoskeletal proteins, including the intermediate filaments desmin and vimentin, has been described in dilated cardiomyopathy, although no data are available from in vivo studies comparing myocardial function in pathological and normal human tissue. ${ }^{10}$ Furthermore, it has been proposed that cytoskeletal injury is associated with irreversible myocardial damage in coronary artery disease. ${ }^{4}$

Desmin mutation has very recently been demonstrated in idiopathic dilated cardiomyopathy and it has been suggested this cytoskeletal protein plays an important functional role in cardiac tissue. ${ }^{11}$ 
Endomyocardial biopsies have in the past yielded conflicting results in dilated cardiomyopathy. Some investigators have found histological changes that appear to be related to left ventricular haemodynamics and prognosis, ${ }^{12}$ while others have failed to show such a correlation. ${ }^{2}{ }^{13}$ Nevertheless, in recent years specimens obtained at endomyocardial biopsy have proved useful in exploring the cellular and molecular abnormalities of the failing human myocardium in vivo. ${ }^{15} 16$

Evaluation of the contractile function of myocytes is very important in heart failure ${ }^{17}$ but difficult to achieve without isolating the cells from the whole heart. ${ }^{18-20}$ The actinmyosin in vitro motility assay is a useful new tool for evaluating actin filament motility on a myosin monomer substrate. ${ }^{21-23}$ This technique provides critical information on the enzymatic and mechanical properties of myosin and could represent a useful approach for evaluating functional sarcomeric alterations in cardiac biopsy tissue.

Our aim in this study was to investigate in vivo-using endomyocardial biopsies from human hearts - the changes in the cytoskeletal intermediate filaments desmin and vimentin that occur in dilated cardiomyopathy in comparison with the normal heart, and to determine whether these alterations are related to cellular contractile dysfunction, as evaluated by the actin-myosin in vitro motility assay.

\section{Methods}

The study protocol was approved by the ethics review committee of the "Federico II" University Medical School in Naples, Italy.

\section{STUDY POPULATION}

The study population consisted of 12 patients with idiopathic dilated cardiomyopathy and 12 controls. The control group comprised six women at high risk of recurrence of breast cancer, who were enrolled in a programme that included endomyocardial biopsy to evaluate possible cardiac toxicity from anthracycline treatment, and six male donors for heart transplantation who had undergone cardiac biopsy at the time of heart explantation. All participants except the donors had been admitted to hospital between January 1994 and July 1998

Table 1 Patient characteristics: clinical and haemodymamic variables

\begin{tabular}{|c|c|c|}
\hline & Controls $(n=12)$ & $D C M \quad(n=12)$ \\
\hline Sex $($ male $/ F)$ & $6 / 6$ & $9 / 3$ \\
\hline Age (years) & $50.14(12.4)$ & $48.4 \quad(13.3)$ \\
\hline Body surface area $\left(\mathrm{m}^{2}\right)$ & $1.74(0.15)$ & $1.89(0.29)$ \\
\hline $\mathrm{SBP}(\mathrm{mm} \mathrm{Hg})$ & 125.3 & $119.4 \quad(11)$ \\
\hline $\mathrm{DBP}(\mathrm{mm} \mathrm{Hg})$ & $81.07(2.12)$ & $79.4 \quad(1.6)$ \\
\hline HR (beats/min) & 85.9 (13) & 83.7 (14) \\
\hline \multicolumn{3}{|l|}{ ECG } \\
\hline Atrial fibrillation & 0 & 4 \\
\hline Left bundle branch block & 0 & 4 \\
\hline ST-T changes & 0 & 9 \\
\hline Left ventricular hypertrophy & 0 & 4 \\
\hline 1 st degree AV block & 0 & 2 \\
\hline Radionuclide ejection fraction (\%) & $64.5(17.6)$ & $34.12(4.11)^{\star}$ \\
\hline Left ventricular mass index $\left(\mathrm{g} / \mathrm{m}^{2}\right)$ & $85.7 \quad(11.8)$ & $187.6(44.3)^{\star}$ \\
\hline
\end{tabular}

Values are mean (SD) or $\mathrm{n}$.

${ }^{\star} \mathrm{p}<0.01$.

$\mathrm{AV}$, atrioventricular; DCM, dilated cardiomyopathy; DBP, diastolic blood pressure; HR, heart rate; SBP, systolic blood pressure. at the emergency medicine division of the department of clinical and experimental medicine ("Federico II" University, Naples, Italy), and all had given written informed consent to be enrolled in the study and to undergo both the invasive and the non-invasive investigations.

All the histological findings obtained at endomyocardial biopsy in the control group reproduced the well known normal ultrastructure of cardiac muscle on light microscopy and electron microscopy. ${ }^{24}{ }^{25}$ The results of immunohistochemical investigations were also considered normal on the basis of the comparison with identical patterns obtained in Sprague-Dawley rats. ${ }^{10}$

The diagnosis of idiopathic dilated cardiomyopathy was made in 12 subjects (table 1 ) on the basis of history, clinical examination (New York Heart Association (NYHA) heart failure score), ECG, chest $x$ ray, Doppler echocardiography, equilibrium radionuclide angiography, thallium tomography, and coronary angiography. The control group, but not the donors, underwent the same investigations apart from the coronary angiography.

\section{CARDIAC BIOPSY}

After isolation of the right internal jugular vein by a 9 French introducer system, a right ventricular cardiac biopsy was carried out. The procedure was performed using a Sholten biotome (8 French) (Sholten, Stanford, California, USA) in the catheter laboratory, with continuous ECG monitoring and under fluoroscopic and echocardiographic control, aiming to take endomyocardial specimens from the middle third of the interventricular septum. Three samples at least were collected from each patient for light microscopy, immunohistochemistry, and actin-myosin in vitro motility assay.

DOPPLER ECHOCARDIOGRAPHIC EVALUATION $M$ mode, cross sectional, and spectral Doppler echocardiographic evaluations were performed using a mechanical ultrasound system equipped with a $3.5 \mathrm{MHz}$ transducer. Recordings were made with the patient in the left lateral position, according to the standardisation recommended by the American Society of Echocardiography.

Measurements included interventricular septal end diastolic dimension, left ventricular posterior end diastolic wall dimension, left ventricular end diastolic dimension, and left ventricular end systolic dimension.

The left ventricular mass was calculated according to Devereux and Reichek. ${ }^{26}$ Left ventricular mass was related to body surface area $\left(\mathrm{LVMi}, \mathrm{g} / \mathrm{m}^{2}\right)$.

RADIONUCLIDE ANGIOGRAPHY

All patients underwent equilibrium radionuclide angiography within one week of echocardiography and thallium imaging, as described by Volpe and colleagues. ${ }^{27}$ In all patients, in vivo labelling of red blood cells was done with $555 \mathrm{Mbq}$ of technetium $\mathrm{Tc}^{99 \mathrm{~m}}$. Radionuclide angiography was performed at 
rest in a $45^{\circ}$ left anterior projection with the patient in the supine position. A small field of view gamma camera (Starcam $300 \mathrm{~A} / \mathrm{M}$, General Electric, Milwaukee, Wisconsin, USA) equipped with a low energy all purpose collimator was used. Data were recorded at a frame rate of 24 frames per cardiac cycle on a dedicated computer system. Radionuclide angiography was analysed using standard software (General Electric).

Reproducibility of ejection fraction measurements in our laboratory has been reported. ${ }^{27}$ In particular, assessment of the ejection fraction within the same patients under steady state conditions on different days of observation showed a significant correlation $(r=0.97$; $\mathrm{p}<0.01$ ), the standard deviation of the reproducibility of the measurements being $1.5 \%$.

HISTOLOGICAL CRITERIA FOR RECRUITMENT

Light microscopic examination excluded the presence of inflammatory cells in the myocardial interstitium, and sites of myocytolysis and contraction band necrosis.

\section{LIGHT MICROSCOPY}

After biopsy, the samples were immediately fixed in phosphate buffered formalin and embedded in paraffin. Serial sections $(4 \mu \mathrm{m}$ thick) were mounted on poly-L-lysine coated slides. About 20 slides were obtained from each block. Non-consecutive sections were stained with haematoxylin and eosin, and picrosirius red. At least five stained slides were used for morphology and stereological evaluation.

\section{IMMUNOCHEMISTRY}

Sections of specimens routinely processed for each case were dewaxed and brought to water. Slides were incubated in $0.1 \%$ sodium azide containing $3 \%$ hydrogen peroxide to quench endogenous peroxidase, then treated with $5 \%$ normal goat serum in phosphate buffered saline (PBS) for 15 minutes to block nonspecific binding.

The primary antibodies were:

- Desmin monoclonal mouse antibody (clone 33, Biogenex, San Ramon, California, USA), at a dilution of $1: 160$;

- Vimentin monoclonal mouse antibody (clone Vim 3B4, Dako A/S, Glostrup, Denmark), at a dilution of 1:50.

The primary antibodies were applied to sections in $1 \%$ bovine serum albumin (BSA), $0.05 \mathrm{M}$ Tris- $\mathrm{HCl}, \mathrm{pH} 7.4$; the slides were then incubated for 60 minutes in a moist chamber at $25^{\circ} \mathrm{C}$.

After three five minute washes in PBS, the slides were treated with secondary biotinylated goat antimouse antibody (Biogenex) at a dilution of 1:50 for 30 minutes.

After three 5 minute washes in PBS, peroxidase conjugated streptavidin (Biogenex) diluted at 1:50 was applied for 45 minutes.

To develop peroxidase activity, the sections were treated for 10 minutes with $0.05 \%$ freshly made and filtered solution of 3-3'diaminobenzidine tetrahydrochloride (DAB) (Sigma Chemical Company, St Louis, Mis- souri, USA) in $10 \mathrm{ml}$ of $0.05 \mathrm{M}$ Tris- $\mathrm{HCl}, \mathrm{pH}$ 7.6 , to which $0.03 \%$ hydrogen peroxide was added.

Negative control sections, to which normal goat serum had been added instead of primary antibody, were included on each slide. Positive control sections were obtained from SpragueDawley rat hearts.

Harris haematoxylin was used as nuclear counterstain, except for sections devoted to densitometric evaluation. ${ }^{28}$

IMAGING, IMAGE ANALYSIS, DATA PROCESSING

Each section was observed with a Zeiss Axioskop photomicroscope, equipped with a stabilised power supply, a $12 \mathrm{~V}, 100 \mathrm{~W}$ tungsten halogen lamp, a 1.4 NA condenser, and a standard set of plan neofluar objectives. Microscopic images were detected with a high resolution CCD digital camera operating on a dynamic range of 12 bits per pixel (Lumina, Leaf Systems Inc, SciTex Europe SA, Waterloo, Belgium), transduced to a PowerMac personal computer (Apple Computers Inc, Cupertino, California, USA) and saved as 8 bit uncompressed files.

Image analysis was performed on monochrome, 256 grey level images, with the public domain NIH Image 1.59 software.

The microscopic images were digitised at a resolution of $600 \mathrm{ppi}$ (corresponding to a final resolution of 2.11 pixels $/ \mu \mathrm{m}$, with the $20 / 0.5$ objective), and then spatially calibrated using images of a standard micrometer object as reference.

For morphometric evaluation, at least three microscopic fields from each haematoxylin and eosin and picrosirius stained section were digitised with the 20/0.5 plan neofluar objective, and serial images were then taken with the $40 / 0.75$ objective. Thus a minimum of 15 fields was taken from each biopsy sample, which corresponded to a tissue area of $3.3 \mathrm{~mm}^{2}$ with the $20 / 0.5$ objective, with a similar number of near longitudinal and near transversely oriented myocytes.

To evaluate the antigen distribution and the immunostain intensity in the tissue, a minimum of three stained sections for each case was observed, and at least 12 microscopic fields with the $\times 20 / 0.5$ objective for each case were digitised and analysed, corresponding to a tissue area of $2.6 \mathrm{~mm}^{2}$.

The guidelines reported by Chieco and colleagues, ${ }^{29}$ related to absorbency image cytometry, were observed in setting up the hardware and in the calibration steps.

After focusing the images, the optical alignment was set directly on the camera viewfinder, and the exposure time was fixed from an examination of a histogram of the grey levels of the image pixels. Thereafter the settings remained constant within each session. Photometric linearity was calibrated with a neutral density step tablet (Kodak) at every session.

A glass interference filter (Oriel Corporation, Stratford, Connecticut, USA; code 53850) transmitting at $480 \mathrm{~nm}$, with a bandwidth of $10 \mathrm{~nm}$ at half peak transmission, was positioned in the field diaphragm plane to 
obtain monochromatic light at a wavelength corresponding to the absorbency maximum of the DAB. ${ }^{30}$

Images of the standard step tablet were taken, and then the integrated grey level values of each step were related to the absorbency values determined spectrophotometrically at the same wavelength by an exponential algorithm.

From each slide, a blank field and a negative control field were digitised at the same time as the fields of interest. The images were density calibrated in optical density units using an external standard technique. Each absorbency calibrated image was processed for shading and background correction by subtracting the absorbency values of the empty reference field.

\section{MORPHOMETRIC EVALUATION}

The sampling methods and stereological formulas applied for calculation were derived as previously described. ${ }^{31-33}$

Estimation of the fractional area $\left(\mathrm{A}_{\mathrm{A}}\right)$, equal to the myocyte fraction volume $\left(\mathrm{V}_{\mathrm{V}}\right)$ in biopsy samples, was performed with a stereological square lattice point grid by counting the fraction of points overlying the myocyte compartments of the tissue. The same method was used to evaluate the percentage of myocardial volume occupied by interstitial tissue. A direct count of the number of nuclear profiles on the area measured was used to calculate the mean number of myocyte nuclei per unit area $\left(\mathrm{mm}^{2}\right)$ of myocytes, $\mathrm{N}(\mathrm{n})_{\mathrm{A}}$. Mean nuclear diameter, D, was calculated from the area of the nuclear profiles, assuming a spherical shape and estimating the particle diameter to be $\mathrm{D}=4 / \pi \mathrm{d}$ (d, profile diameter). ${ }^{32}$ Myocyte diameter was measured orthogonal to cell profiles and estimated to be $\mathrm{D}=4 / \pi \mathrm{d}$, assuming a cylindrical shape ( $\mathrm{d}$, profile diameter). Owing to the small number of longitudinally sectioned myocyte nuclei it was necessary to have both diameter estimates.

EVALUATION OF IMMUNOSTAINING

Antigen distribution was evaluated on density calibrated monochrome images. Each image was converted in pseudocolours by fixing the colour palette at the staining intensity of the tissue background. All the points of a morphometric grid overlying the stained regions of the myocyte compartment were counted. This point fraction was related to the points of the overall myocyte compartment, and expressed as per cent staining of myocytic cytoplasm.

Staining intensity was measured on the same compartment as absorbency at $480 \mathrm{~nm}$ of image pixels. The regions of interest were defined by thresholding and segmentation. The mean optical density of myocyte cytoplasm was computed as the arithmetic mean of the absorbency values of all the pixels. This variable is related to the mean antigen concentration. Absorbency values were corrected for the blank fields by subtracting the mean absorbency of corresponding areas from a negative control section. The average integrated absorbency of myocyte average area per cell nucleus was calculated from the sum of the absorbency values of all the pixels covering the myocyte unit area and the mean area of myocytes in each section, derived from the number of nuclear profiles per unit area.

The results for each individual case were the average of values obtained from each image processed. The results are presented as mean (SD) of all the cases included in the study group.

\section{TEST SPECIMENS}

A test system was designed and evaluated as described by Huang and colleagues, ${ }^{34}$ to assess the reliability of densitometry for detecting changes in tissue antigen content as a function of staining intensity.

To mimic the changes in antigen concentration, rat hearts were dissected free from the atria and homogenised on ice. Tissue homogenate was mixed with tissue embedding compound (OCT, Miles Scientific, Elkhart, Indiana, USA) in serial ratios from $100 \%$ to $50 \%$ (vol/vol) of tissue to OCT compound. The samples were frozen in plastic tubes at $-80^{\circ} \mathrm{C}$; $4 \mu \mathrm{m}$ sections were then cut with a Leitz cryostat at $-20^{\circ} \mathrm{C}$ and thaw mounted onto slides. Tissue disks thus underwent standard immunochemical procedures. Immunostaining was evaluated on calibrated images taken from 15 stained tissue disks for each dilution, as described in the previous section. On test disks stained with desmin monoclonal antibody, both mean optical density at $480 \mathrm{~nm}$ and the integrated density per $\mathrm{mm}^{2}$ show a linear positive relation with the increase in the tissue content of the homogenate $(p<0.001)$.

A positive linear relation $(\mathrm{p}<0.01)$ was also shown between the same variables and section thickness in the range 3-8 $\mu \mathrm{m}$, on images taken from routinely fixed, paraffin embedded, and immunostained slides (five stained tissue sections for each level of thickness).

ACTIN-MYOSIN IN VITRO MOTILITY ASSAY

Myocardial biopsies from patients affected by dilated cardiomyopathy and from control patients were used to study the pathophysiology of myosin in an in vitro motility assay. ${ }^{35}$ This assay measures the sliding rate of rhodamine phalloidin labelled actin filaments translocated by myosin molecules bound to a nitrocellulose coated surface.

\section{Purification of proteins}

Myosin was purified from cardiac biopsies as previously described. ${ }^{21}$ Briefly, frozen tissue was pulverised and washed three times in five volumes of buffer A containing $20 \mathrm{mM}$ Mops (pH 7.0), $40 \mathrm{mM} \mathrm{KCl,} 5 \mathrm{mM}$ EGTA, $2 \mathrm{mM}$ DTT, $0.01 \% \mathrm{NaN}_{3}, 0.1 \mathrm{mM}$ PMSF, $10 \mathrm{mg} / \mathrm{l}$ pepstatin, $10 \mathrm{mg} / 1$ tosyl-L-phenylalanine chloromethyl ketone, $10 \mathrm{mg} / \mathrm{l}$ leupeptin, $5 \mathrm{mg} / 1$ chymostatin, and $10 \mathrm{mg} / 1$ tosyl-L-lysine chloromethyl ketone. To purify myosin, the washed myofibrils were resuspended in buffer $\mathrm{A}$ and extracted for 15 minutes by the addition of $\mathrm{NaCl}, \mathrm{ATP}$, and $\mathrm{MgCl}_{2}$ to final concentrations of $0.5 \mathrm{M}, 10 \mathrm{mM}$, and $10 \mathrm{mM}$, respectively. The sample was allowed to sediment for 15 minutes at $470000 \mathrm{~g}$, using a TL 100 ultracen- 
trifuge (Beckmann Instruments Inc, Palo Alto, California, USA).

Rabbit skeletal muscle actin was prepared as described previously. ${ }^{36}$

In vitro motility assay

Pure cardiac myosin was bound to the nitrocellulose coated surface of the flow cell in monomeric form, as described by Umemoto and Sellers. ${ }^{23}$

The flow cell was filled with $0.5 \mathrm{mg} / 1 \mathrm{BSA}$ in buffer $\mathrm{B}$, containing $0.5 \mathrm{M} \mathrm{NaCl}, 10 \mathrm{mM}$ Mops ( $\mathrm{pH} 7.0$ ), $0.1 \mathrm{mM}$ EGTA, and $1 \mathrm{mM}$ DTT. After one minute, cardiac myosin was added to buffer B. After five minutes, the same buffer was used to remove the unbound material.

In other respects the in vitro motility assay was performed and the results quantified as described by Homsher and colleagues. ${ }^{37}$

The composition of the assay buffer was $20 \mathrm{mM} \mathrm{KCl}, 10 \mathrm{mM}$ Mops (pH 7.2), $5 \mathrm{mM}$ $\mathrm{MgCl}_{2}, 1 \mathrm{mM}$ ATP, $0.1 \mathrm{mM}$ EGTA, $10 \mathrm{mM}$ DTT, $0.7 \%$ methyl cellulose, $2.5 \mathrm{mg} / \mathrm{ml}$ glucose, $0.1 \mathrm{mg} / \mathrm{ml}$ glucose oxidase, and $0.02 \mathrm{mg} /$ $\mathrm{ml}$ catalase.

Analysis of the sliding movements was performed with a video based system (Celltrack; Motion Analysis, Santa Rosa, California, USA).

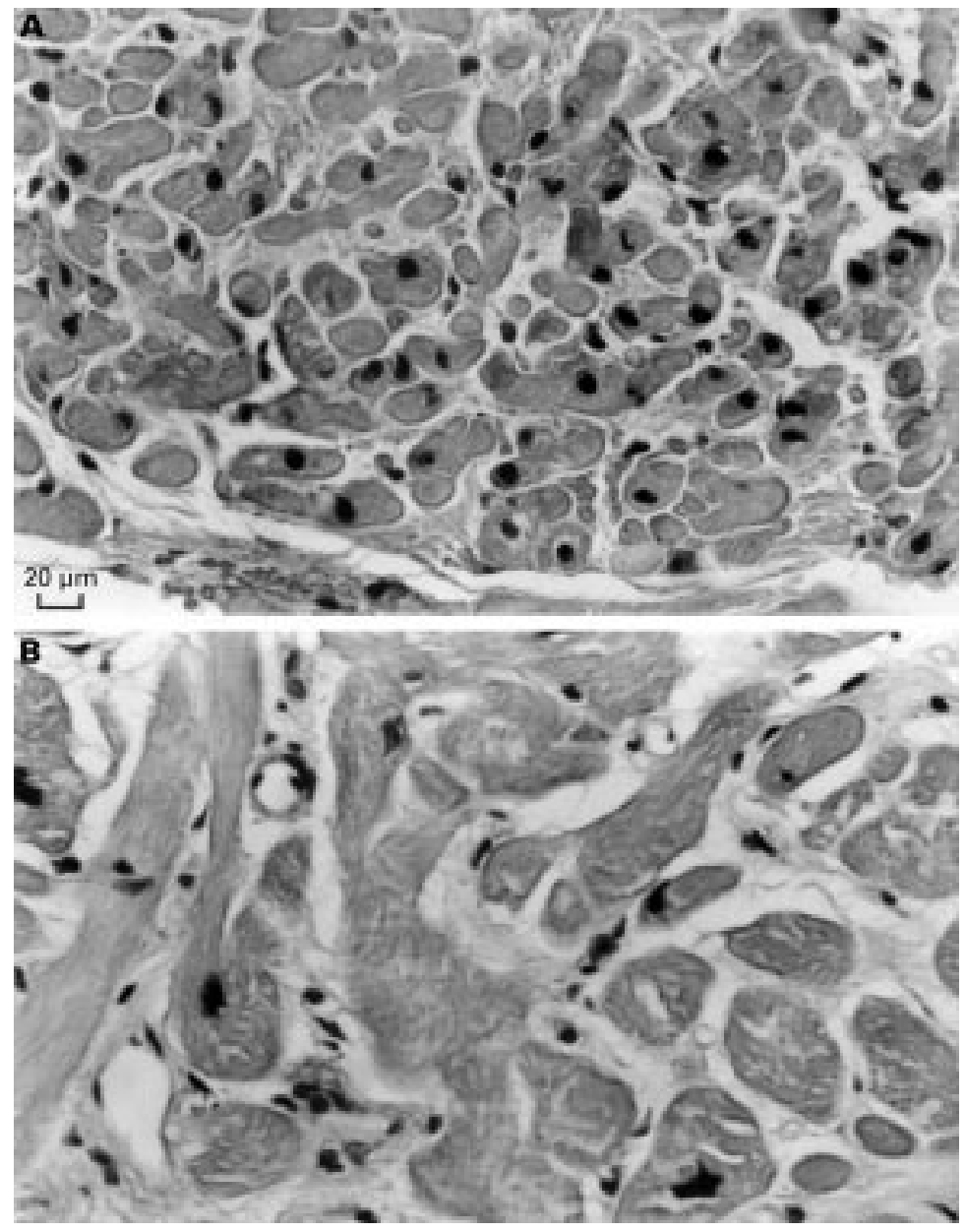

Figure 1 (A) Myocardial tissue from control patient (haematoxylin and eosin, $\times 40$ ). (B) Myocardial tissue from patient with dilated cardiomyopathy (haematoxylin and eosin, $\times 40)$.
STATISTICAL ANALYSIS

All data were collected blind. Results are shown as mean (SD) of $\mathrm{n}$ cases in the study groups, obtained from the average measurements of each biopsy sample. Comparisons between means were performed by the nonparametric Wilcoxon two sample test. Correlation coefficients between each set of data were determined by the Spearman rank regression analysis. Probability values of $p<0.05$ were considered significant.

\section{Results}

The two groups of patients were comparable for sex, age, body surface area, blood pressure, and heart rate (table 1 ).

Among the 12 patients with dilated cardiomyopathy, 10 were in NYHA class III and two were in class IV. All patients were being treated with digitalis, diuretics, and angiotensin converting enzyme (ACE) inhibitors. On ECG, four patients had atrial fibrillation, four had complete left bundle branch block, nine had ST-T changes, four had left ventricular hypertrophy, and two had first degree atrioventricular block.

Coronary angiography excluded the presence of significant coronary lesions in all patients with dilated cardiomyopathy.

At radionuclide angiography, the left ventricular ejection fraction was lower $(p<0.01)$ in the cardiomyopathy group than in the control group (table 1).

\section{ECHOCARDIOGRAM}

In the cardiomyopathy group there was a 2.6-fold increase in left ventricular mass compared with the control group; the left ventricular mass to body surface area ratio was increased by $115 \%$ (table 1 ).

The transverse left ventricular chamber diameter at the end diastole was increased by $47 \%$, and the computed chamber volume expanded by 4.1 -fold; this reduced both the left ventricular mass to left ventricular volume ratio and the left ventricular wall thickness to chamber radius ratio.

\section{LIGHT MICROSCOPY}

On light microscopy, the right ventricular endomyocardial biopsies showed hypertrophic changes in the myocytes with nuclear enlargement (fig 1B) in the patients with cardiomyopathy but not in the controls (fig 1A). In the cardiomyopathy group, the interstitial compartment appeared expanded, mainly because of interstitial fibrosis (fig 1B). Morphological data are summarised in table 2 .

The myocyte area was similar in the cardiomyopathy patients and the controls; collagen fibres showed a 0.4-fold increase in the cardiomyopathy group, but this was not significant.

The number of myocyte nuclei per unit area of myocytes was reduced by $41 \%(\mathrm{p}<0.001)$ in the cardiomyopathy group compared with the controls (table 2). The average myocyte nuclear area in the cardiomyopathy group was increased by $59 \%$ compared with the controls $(\mathrm{p}<0.005)$ 
Table 2 Morphometric evaluation of myocardial tissue on light microscopy (haematoxylin and eosin, and picrosirius red)

\begin{tabular}{lccl}
\hline & Controls $(n=12)$ & DCM $(n=12)$ & $p$ Value \\
\hline $\begin{array}{l}\text { Fractional area }(\%) \text { of } \\
\text { myocardial tissue }\end{array}$ & $83.65(5.04)$ & $82.93(6.33)$ & NS \\
$\begin{array}{l}\text { Number of nuclei/mm } \text { of }^{2} \\
\quad \text { myocardial tissue }\end{array}$ & $668.22(131.29)$ & $392.83(71.78)$ & 0.001 \\
Nucleus diameter $(\mu \mathrm{m})$ & $8.35(1.06)$ & $10.45(1.98)$ & 0.01 \\
Nucleus area $\left(\mu \mathrm{m}^{2}\right)$ & $55.56(14.08)$ & $88.27(33.12)$ & 0.005 \\
Myocyte diameter $(\mu \mathrm{m})$ & $16.74(1.17)$ & $22.89(3.30)$ & 0.002 \\
Fibrosis area $(\%)$ & $13.39(9.55)$ & $18.71(7.76)$ & NS
\end{tabular}

Values are mean (SD) or $\mathrm{n}$.

DCM, dilated cardiomyopathy.

Myocyte diameter was increased by $37 \%$ in the cardiomyopathy group compared with the controls $(\mathrm{p}<0.002)$ (table 2$)$.

\section{IMMUNOCYTOCHEMISTRY}

\section{Desmin}

In cardiac myocytes from the control group, immunostaining to desmin was localised to the $\mathrm{Z}$ lines, resulting in a regular pattern of cross striation (fig 2A). Furthermore, in many cells an increase in desmin staining at the cell ends labelled the intercalated disks. In myocytes from the cardiomyopathy patients, an irregular

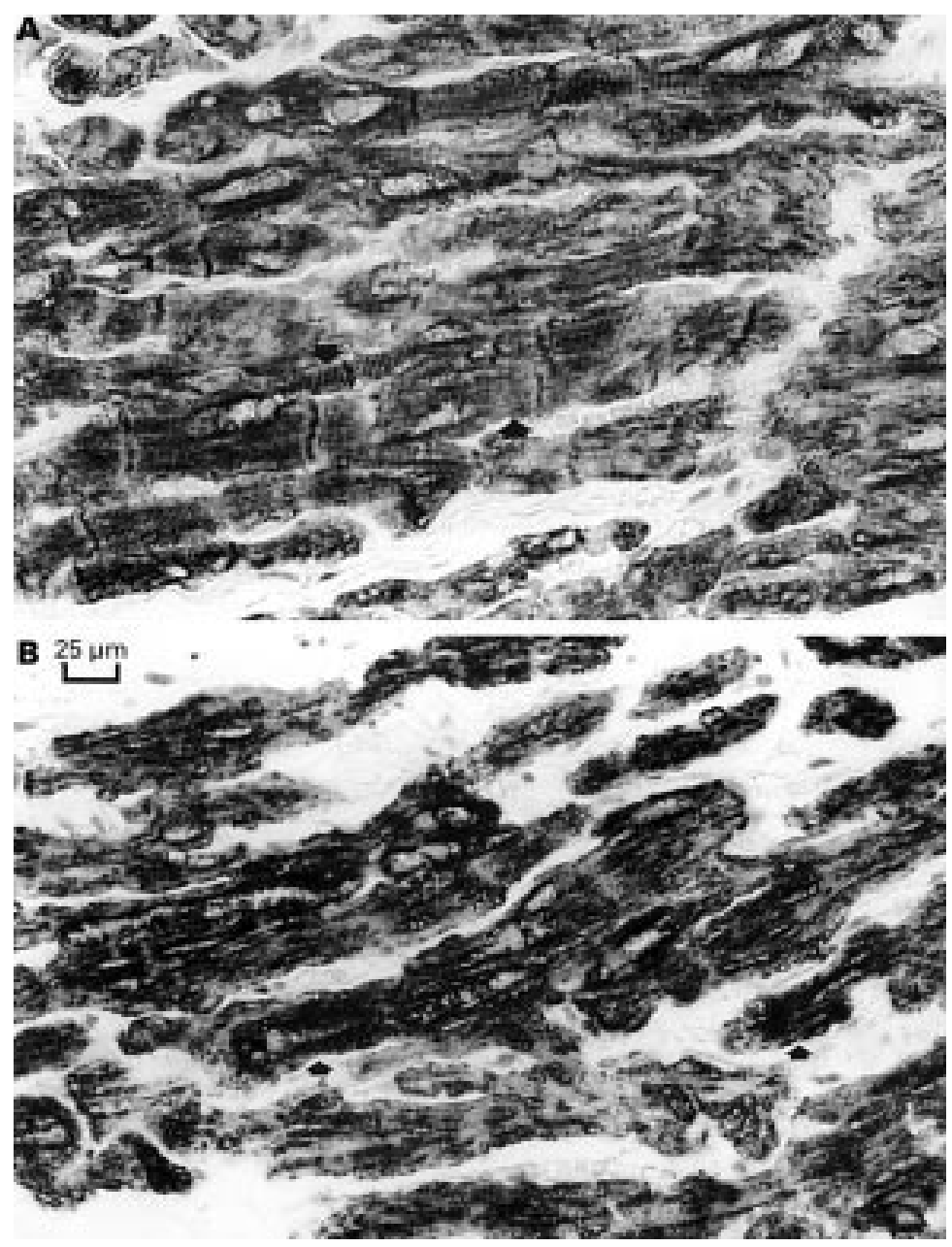

Figure 2 (A) Immunostaining by desmin monoclonal antibody in myocardium of a control patient. The arrows indicate the regular pattern of cross striation and the $Z$ lines ( $\times 40)$. (B) Immunostaining by desmin monoclonal antibody in myocardium of a patient with dilatated cardiomyopathy. The arrows indicate the irregular pattern of desmin staining giving a scattered appearance, with areas of increased staining and areas of no staining $(\times 40)$. The photomicrographs were obtained using an interference filter transmitting at $480 \mathrm{~nm}$ to enhance the diaminobenzidine hydrochloride (DAB) contrast. pattern of desmin staining resulted in a scattered appearance (fig 2B). Cell staining was reduced or absent in some cytoplasmic regions, contrasting with the pronounced staining in both the perinuclear region and intermyofibrillar spaces, where clumps of filaments were present (fig 2B).

When the staining distribution was evaluated by morphometric sampling, the proportion of the myocyte cytoplasm area reacting with monoclonal desmin antibody was $73 \%$ in the control group and $52 \%$ in cardiomyopathy group $(-29 \% ; \mathrm{p}<0.002)$.

When the desmin content was evaluated by image densitometry, the mean optical density of myocyte cytoplasm, which is related to the mean antigen concentration, was not significantly different from control $(0.22$ in controls $v$ 0.24 in cardiomyopathy patients). However, the integrated absorbency per average area myocyte profile, which is related to the mean amount of antigen, was increased by $55 \%$ $(p<0.05)$, from 406 in the controls to 629 in the cardiomyopathy group. This increase was related to the increased cell dimensions in the cardiomyopathy patients, as shown by the linear relation between integrated absorbency of desmin and myocyte diameter $(r=0.9418$; $\mathrm{p}<0.001$ ).

In summary, in dilated cardiomyopathy the myocyte content of desmin intermediate filaments was greater than in the controls, and the distribution was irregular.

There was a negative correlation between the amount of myocardial desmin and the ejection fraction $(r=-0.773$; p < 0.02) (fig 3), whereas there was a positive relation between desmin distribution and the ejection fraction $(r=0.764 ; \mathrm{p}<0.02)$ and the actin-myosin in vitro motility assay $(r=0.853 ; \mathrm{p}<0.01)$ (fig 4). A positive linear correlation was also present between desmin content and left ventricular mass as determined by echocardiography $(r=0.7 ; \mathrm{p}<0.05)$.

\section{Vimentin}

In both groups, vimentin staining labelled the cells of the interstitial space (fibroblasts, endothelial cells, and vascular smooth muscle cells; fig 5, A and B).

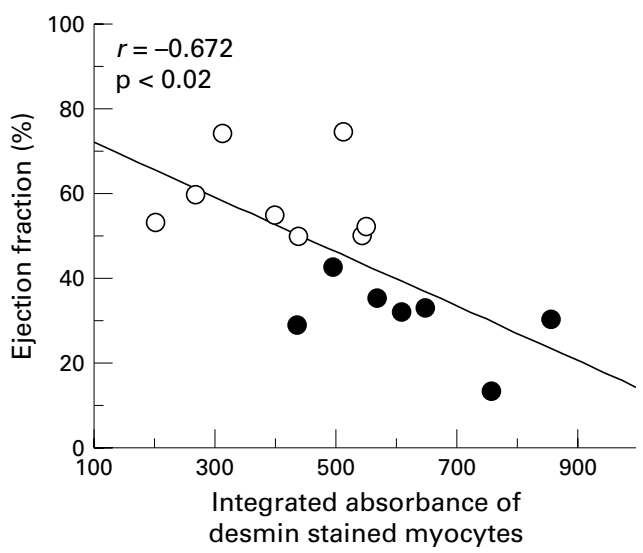

Figure 3 Negative relation between myocyte desmin stained integrated absorbance and ejection fraction determined by radionuclide angiography. Empty circles, controls; filled circles, patients with dilated cardiomyopathy. 


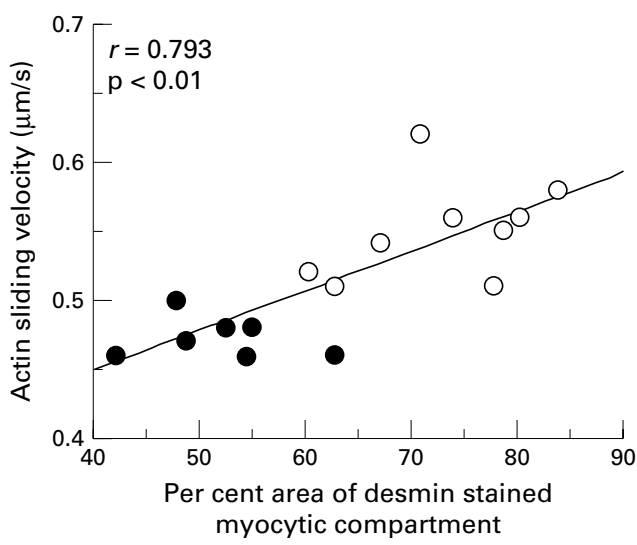

Figure 4 Linear relation between myocardial desmin distribution and actin-myosin in vitro motility assay. Empty circles, controls; filled circles, patients with dilated cardiomyopathy.

When myocardial vimentin content was evaluated by morphometric sampling, a mean of $4.9 \%$ of the area of cardiac tissue reacted with monoclonal vimentin antibody in the control group (fig $5 \mathrm{~A}$ ) and $11.4 \%$ in the cardiomyopathy group (fig 5B) (+132.8\%; $\mathrm{p}<0.05)$. There was a negative correlation between vimentin content and the ejection
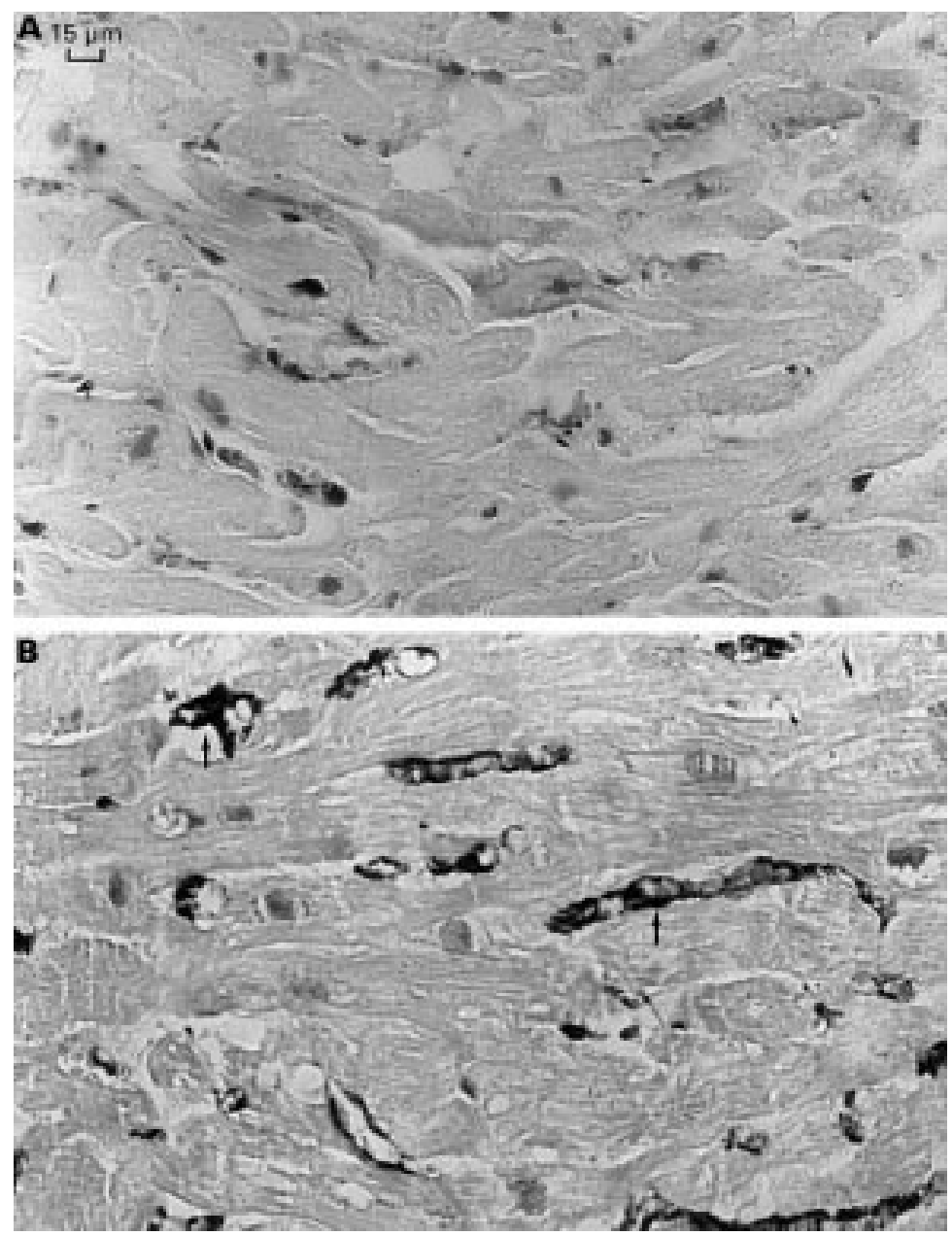

Figure 5 (A) Immunostaining by vimentin monoclonal antibody on myocardium of a control patient $(\times 40)$. (B) Immunostaining by vimentin monoclonal antibody on myocardium of a patient with dilatated cardiomyopathy $(\times 40)$. The arrow indicates the increase in vimentin staining in the interstitial space. The photomicrographs were obtained using an interference filter transmitting at $480 \mathrm{~nm}$ to enhance the DAB contrast. fraction $(r=-0.68 ; \mathrm{p}<0.01)$ and the actinmyosin sliding rate $(\mathrm{r}=-0.74, \mathrm{p}<0.02)$, whereas there was a positive correlation between vimentin content and left ventricular mass $(r=0.656 ; \mathrm{p}<0.02)$. There was also a positive correlation between the proportion of vimentin stained cells and fibrosis $(r=0.68$; $\mathrm{p}<0.05)$.

ACTIN-MYOSIN IN VITRO MOTILITY ASSAY Biopsies from the controls and the cardiomyopathy patients were used to study the functional properties of purified myosin in an in vitro motility assay, where labelled actin filaments were translocated by myosin bound to a coverslip surface.

Myosin from control hearts translocated actin filaments at a mean (SD) rate of 0.55 (0.032) $\mu \mathrm{m} / \mathrm{s}$. The actin sliding velocity in the patients with cardiomyopathy was only 0.47 $(0.015) \mu \mathrm{m} / \mathrm{s}(\mathrm{p}<0.05)$.

There was a significant negative correlation between actin sliding rate and both myocyte diameter $(r=-0.642 ; \mathrm{p}<0.01)$ and left ventricular mass on echocardiographic evaluation $(r=-0.788 ; \mathrm{p}<0.01)$. There was a significant positive correlation between actin sliding rate and the ejection fraction $(r=0.8 ; \mathrm{p}<0.002)$.

\section{Discussion}

The main histological finding in our study was an increase in myocyte dimensions in the biopsy samples from the patients with dilated cardiomyopathy, as shown by the significant difference in myocyte diameter value on morphometric evaluation by light microscopy. This morphological change is in agreement with previous observations ${ }^{12}$ and has been attributed to compensatory cell growth in response to volume overload. However, the increased myocyte growth did not appear to compensate for impaired cellular function-in fact we found a negative relation between myocyte diameter and contractile protein motility, implying that the larger the cell the less capable it is of contracting. This was confirmed by the fact that the actin-myosin sliding rate was significantly reduced in the cardiomyopathy group compared with the controls, and was also inversely related to left ventricular mass on echocardiographic evaluation. Moreover there was a linear correlation between the ejection fraction and the actin-myosin motility assay, indicating that this in vitro index was related to left ventricular function of the whole heart in vivo.

In our view these findings have considerable clinical relevance in that the disturbance of actin-myosin in vitro motility in cardiac biopsies from patients with dilated cardiomyopathy could form the basis for a prognostic test in these patients.

Our results show a significant increase in vimentin stained cells in cardiomyopathy patients compared with the controls, which has been linked to an increase in interstitial heart tissue by other investigators. ${ }^{10}$ In support of this hypothesis, we found a positive correlation between vimentin positive cells and fibrosis, indicating an increase in interstitial tissue in 
this area. Therefore, although fibrotic tissue may not always be increased in dilated cardiomyopathy, ${ }^{12}$ an increase in fibroblast vimentin could be a precursor of fibrosis.

A computer evaluation of the desmin content in biopsy samples showed a significant increase in the amount of desmin in diseased myocardial cells compared with normal tissue. This confirms the data obtained by Schaper and colleagues in dilated cardiomyopathy. ${ }^{10}$ However, in that study the results were obtained in explanted hearts, and the control group consisted of myocardium from rats and pigs, whereas we used human myocardial tissue from endomyocardial biopsies, which allowed us to obtain in vivo results. We also described areas of desmin accumulation, as did Schaper. ${ }^{10}$ Moreover, our computer evaluation indicated that the desmin distribution was uneven in the biopsies from the patients with cardiomyopathy, and we speculate that in this disorder the increase in cell dimensions leads not only to an increased desmin content but also to irregular desmin distribution.

In normal hearts, desmin forms an interconnected network which, through its longitudinal and transverse elements, keeps the myofibrils at the $\mathrm{Z}$ disk in axial orientation. ${ }^{4}$ Morphological studies have shown that desmin is strictly related to the sarcomere at the $\mathrm{Z}$ disks, and that the intermediate filaments play a regulatory role $^{45}$ on the normal contractile function of the myofibrils. In other words, we can assume that there is a myofibril-desmin relation in the sarcomere, the integrity of which is essential for the normal contraction of the myocyte. This hypothesis is strengthened in our study by the positive correlation between desmin distribution and the contractile protein coupling rate. It has been reported that cytoskeletal alterations are responsible for the cellular contractile dysfunction in cardiac hypertrophy in response to pressure loading ${ }^{38-40}$; persistence of this alteration could be a significant factor in the deterioration of the initially compensatory cellular mechanisms, leading eventually to heart failure. $^{7}$

Studies of the intermediate filaments in hypertrophied myocardium have shown an increase in the amount of desmin, in an attempt to maintain the newly formed sarcomeres aligned and to keep the myofibrils in register during the contraction-relaxation process. ${ }^{38}$ Moreover, Collins and colleagues have shown a further increase in desmin in the transition from compensated left ventricular hypertrophy to heart failure. ${ }^{8}$ The importance of an even distribution of desmin for normal heart function is also suggested by the significant relation between desmin distribution and left ventricular ejection fraction found in our data. In other words, it seems that in myocytes from patients with dilated cardiomyopathy the increase in cellular desmin content and the irregular desmin distribution are capable of hampering normal contractile function.

An increase in the other myocardial intermediate filament, vimentin, also seems to contribute to the deterioration in left ventricular function in patients with dilated cardiomyopathy.
Our data indicate that an increase in the vimentin content of tissue interstitial cells in these patients was directly correlated with increased left ventricular mass and impairment of the ejection fraction.

Our finding of a positive relation between desmin distribution and left ventricular ejection fraction, and a negative relation between myocardial vimentin content and left ventricular dimensions and function, emphasises the pathophysiological significance of alterations to the intermediate filaments in dilated cardiomyopathy. We speculate that the severity of intermediate filament involvement in cardiac tissues may serve as a marker of prognosis in this condition, and endomyocardial biopsy could represent a useful tool for monitoring progress.

\section{CONCLUSIONS}

The changes in intermediate cytoskeletal filaments observed in myocardial tissue from cardiac biopsies in patients with dilated cardiomyopathy are characterised by an increase in desmin and vimentin content in comparison with normal hearts. This may represent a late stage in the body's attempt to compensate for inadequate cardiac function by replacing the contractile material. ${ }^{10}$

An increase in the size of the cardiomyocytes and a reduction in their numbers, combined with desmin disarray and an increase in vimentin positive cells, are reflected in a decreased ejection fraction and a reduction in the actin-myosin coupling rate, resulting in the inability of the myocyte to achieve adequate contraction.

The clinical implications of our study are that evaluation of the integrity of intermediate filaments in myocytes obtained in vivo by cardiac biopsy could be a marker of the severity of cellular dysfunction in patients with dilated cardiomyopathy. Desmin disarray may represent a late stage of myocyte degeneration, reflected in a failure to achieve adequate myocyte contraction.

A future therapeutic target is to preserve the integrity of cytoskeletal intermediate filaments in order to postpone or prevent the development of irreversible myocyte damage. Evaluation of desmin and vimentin in cardiac biopsies could be helpful in the decision making process in patients on the waiting list for heart transplantation.

This study was supported by Italian National Research Council, Rome, Italy.

1 Beltrami CA, Finato N, Rocco M, et al. The cellular basis of dilated cardiomyopathy in humans. $\mathcal{F} \mathrm{Mol}$ Cell Cardiol 1995;27:291-306.

2 Baandrup U, Florio RA, Roters F, et al. Electron microscopic investigation of endomyocardial biopsy samples in hypertrophy and cardiomyopathy: a semiquantitative study in 48 patients. Circulation 1981;63:1289-98.

3 Gallo P, d'Amati G, Pelliccia F, et al. Functional significance of myocellular hypertrophy in dilated cardiomyopathy: histomorphometric analysis on 40 endomyocardial biopsies. Am $\mathcal{F}$ Cardiovasc Pathol 1995;5:11-18.

4 Ganote C, Armstrong S. Ischaemia and the myocyte cytoskeleton: review and speculation. Cardiovasc Res 1993; 27:1387-403.

5 Capetanaki YG, Ngai J, Lazarides E. Characterization and regulation in the expression of a gene coding for the intermediate filament protein desmin. Proc Natl Acad Sci USA 1984;81:6909-13. 
6 Bristow MR, Gilbert EM. Improvement in cardiac myocyte function by biological effects of medical therapy: a new concept in the treat

7 Tagawa H, Rozich JD, Tsutsui H, et al. Basis of increased microtubules in pressure-hypertrophied cardiocytes. Circu lation 1996;93:1230-43.

8 Collins IF, Pawloski-Dabree C, Davis MG, et al. The role of cytoskeleton in left ventricular pressure overload. Hypertrophy and failure. $7 \mathrm{Mol} \mathrm{Cell} \mathrm{Cardiol} \mathrm{1996;28:1435-43.}$

9 Price MG. Molecular analysis of intermediate filament cytoskeleton-a putative load-bearing structure. Am $\mathcal{F}$
Physiol 1984;246:H566-72.

10 Schaper J, Froede TA, Hein St, et al. Impairment of the myocardial ultrastructure and changes of the cytoskeleton in dilated cardiomyopathy. Circulation 1991;83:504-14.

$11 \mathrm{Li} \mathrm{D}$, Tapscoft T, Gonzales $\mathrm{O}$, et al. Desmin mutation responsible for idiopathic dilated cardiomyopathy. Circulation 1999;100:461-4.

12 Nakayama Y, Shimizu G, Hirota Y, et al. Functional and histopathologic correlation in patients with dilated cardiomyopathy: an integrated evaluation
analysis. Am 7 Cardiol 1987;10:186-92.

13 Figulla H, Rahlf G, Nieger M, et al. Spontaneous haemodynamic improvement or stabilization and associated biopsy findings in patients with congestive cardiomyopathy. Circulation 1985;71:1095-104.

14 Keren A, Gottlieb S, Tzivoni D, et al. Mildly dilated congestive cardiomyopathy. Use of prospective diagnostic criteria and description of the clinical course without heart transplantation. Circulation 1990;81:506-17.

15 Gauthier C, Laurent K, Charpentier F, et al. Endomyocardial biopsies: a new approach for studying the electrical and mechanical properties of human ventricular myocardium. $\mathcal{F}$ Mol Cell Cardiol 1994;26:1267-71.

16 Figueredo VM, Camacho SA. Basic mechanism of myocardial dysfunction: cellular pathophysiology of heart failure. Curr Opin Cardiol 1995;10:246-52.

17 Schwinger RHG, Bohm M, Koch A, et al. The failing human heart is unable to use the Frank-Starling mechhuman heart is unable to use

18 Anversa P, Di Somma S, Bianchi G, et al. Cellular mechanism of cardiac failure in the infarcted heart. Cardio mechanism of cardiac

19 Capasso JM, Anversa P. Mechanical performance of spared myocytes after myocardial infarction in rats: effects of captopril treatment. Am F Physiol 1992;263(Heart Circ Physio 32): H841-9.

20 Wood DS, Zolman JR, Reuben P, et al. Human skeletal muscle: properties of the chemically skinned fiber. Science 1975;187:1075-86.

21 Cuda G, Fanapazir L, Zhu WS, et al. Skeletal muscle expression and abnormal function of beta-myosin in hypertrophic cardiomyopathy. $\mathcal{F}$ Clin Invest 1993;91:2861-5.

22 Sellers JR, Cuda G, Wang F, et al. Myosin specific adaptations of the motility assay. Methods Cell Biol 1993;39: $23-49$.

23 Unemoto S, Sellers JR. Characterization of in vitro motility assay using smooth muscle and cytoplasmic myosin. $\mathcal{F}$ Biol assay using smooth muscle
24 Unverferth DV, Fetters JK, Unverferth BJ, et al. Human myocardial histologic characteristics in congestive heart myocardial histologic characteristics

25 Unverferth DV, Magorien RD, Unverferth BP, et al. Human myocardial morphologic and functional changes in the first 24 hours after doxorubicin administration. Cancer Treat Rep 1981;65:1093-100.

26 Devereux RB, Reichek N. Echocardiographic determination of left ventricular mass in man: anatomic validation of method. Circulation 1977;5:613-18.

27 Volpe M, Rao MAE, Cuocolo A, et al. Radionuclide monitoring of cardiac adaptations to volume loading in patients with dilated cardiomyopathy and mild heart failure. Circulation 1995;92:2511-18.

28 Thal DR, Horn M, Schlote W. Selective quantitative analysis of the intensity of immunohistochemical reactions. Acta Histochem 1995;97:203-11.

29 Chieco P, Jonker A, Melchiorri C, et al. A user's guide for avoiding errors in absorbency image cytometry: a review with original experimental observations. Histochem $\mathcal{f} 1994$; 26:1-19.

30 Frasch ACC, Itoiz ME, Cabrini RL. Microspectrophotometric quantitation of the diaminobenzidine reaction for histochemical demonstration of cytochrome oxidase activity. F Histochem 1978;26:157-62.

31 Linzbach AJ. Heart failure from the point of view of quantitative anatomy. Am $\mathcal{F}$ Cardiol 1960;5:370-82.

32 Olivetti G, Anversa P, Loud AJ. Morphometric study of early postnatal development in the left and right ventricular myocardium of the rat. II. Tissue composition, capillary growth and sarcoplasmic alterations. Circ Res 1980;46:503-

33 Weibel ER, Bolender RP. Stereological technique for electron microscopic morphometry. In: Hayat MA, ed. Principles and techniques of electron microscopy, vol 3. New York: Von Nostrand Reinhold Co, 1973:239.

34 Huang X, Chen S, Tietz EI. Immunocytochemical detection of regional protein changes in rat brain sections using impersis. 7 Histochem Cytochem 1996;44:981-7.

35 Kron SJ, Toyoshima YY, Uyeda TOP, et al. Assays for actin sliding movement over myosin-coated surfaces. Methods Enzymol 1991;196:399-416.

36 Eisenberg E, Keilley WW. Troponin-tropomyosin complex.

37 Homsher EF, Wang F, Sellers JR. Factors affecting movement of F-actin filaments propelled by skeletal muscle heavy meromyosin. Am f Physiol 1992;262(Cell Physiol 31):C714-23.

38 Watkins SC, Samuel JL, Marotte B, et al. Microtubules and desmin filaments during onset of heart hypertrophy inn rat: a double immunoelectron microscope study. Circ Res 1987; 60:327-36.

39 Tsutsui H, Ishihara K, Cooper G. Cytoskeletal role in the contractile dysfunction of hypertrophied myocardium. Science 1993;260:682-7.

40 Tsusti H, Hirifumi T, Kent RL, et al. Role of microtubules in contractile dysfunction of hypertrophied cardiocytes. in contractile dysfunction of 